\title{
The Up-Regulation of miR-199b-5p in Erythroid Differentiation Is Associated with GATA-1 and NF-E2
}

\author{
Yuxia Li ${ }^{1,2}$, Hua Bai ${ }^{3}$, Zhongzu Zhang ${ }^{1}$, Weihua $\mathrm{Ii}^{4}$, Lei Dong ${ }^{1}$, Xueju Wei ${ }^{1,2}$, Yanni Ma ${ }^{1}$, Junwu Zhang ${ }^{1}$, \\ Jia $\mathrm{Yu}^{1}$, Guotao Sun ${ }^{2, *}$, and Fang Wang ${ }^{1, *}$
}

\begin{abstract}
MicroRNAs (miRNAs) represent a class of small non-coding regulatory RNAs that play important roles in normal hematopoiesis, including erythropoiesis. Although studies have identified several miRNAs that regulate erythroid commitment and differentiation, we do not understand the mechanism by which the crucial erythroid transcription factors, GATA-1and NF-E2 directly regulate and control differentiation via miRNA pathways. In this study, we identified miR-199b-5p as a key regulator of human erythropoiesis, and its expression was up-regulated during the erythroid differentiation of K562 cells. Furthermore, the increase of miR-199b-5p in erythroid cells occurred in a GATA-1- and NF-E2-dependent manner during erythrocyte maturation. Both GATA-1 and NF-E2 bound upstream of the miR-199b gene locus and activated its transcription. Forced expression of miRNA-199b-5p in K562 cells affected erythroid cell proliferation and maturation. Moreover, we identified c-Kit as a direct target of miR-199b-5p in erythroid cells. Taken together, our results establish a functional link among the erythroid transcription factors GATA-1/NF-E2, miR-199b-5p and c-Kit, and provide new insights into the coupling of transcription and posttranscription regulation in erythroid differentiation.
\end{abstract}

\section{INTRODUCTION}

Hematopoiesis is the process by which all of the distinct cell lineages that form the blood and immune systems are generated from a common pluripotent stem cell type (Huang et al., 2011). Normal erythropoiesis in adult humans generates approximately $10^{11}$ new red blood cells each day via the commitment of hematopoietic stem cells into erythroid progenitors, which subsequently differentiate into mature cells (Wang et al., 2011). Although hematopoiesis involves a variety of modulators that are influenced by each other in a complex manner, the central role of transcription factors in this bioprocess has been highlighted to date (Fukao et al., 2007).

For example, several critical transcriptional factors, such as GATA-1 and NF-E2, are indispensable for erythropoiesis, because they regulate the expression of multiple erythroid genes (Zhang et al., 2010).

MicroRNAs (miRNAs) are endogenous single-stranded noncoding RNA molecules of 19-25 nucleotides that control gene expression primarily at the post-transcriptional level by binding to the 3'untraslated region (UTR) of target mRNAs to regulate their stability and translation (Faraoni et al., 2012). For example, microRNA-96 directly suppresses gamma-globin expression and contributes to $\mathrm{HbF}$ regulation (Azzouzi and Wollscheid, 2011). miR-150 is up-regulated during $B$ and $T$ cell maturation (Navarro and Lieberman, 2010).

miR-451 is required for zebrafish and mouse erythroid development (Pase et al., 2009; Yu et al., 2010). miR-125b overexpression causes leukemia in mice (Bousqueta et al., 2010). miR-223 has been shown to influence granulocytic differentiation (Bellon et al., 2009). miR-126 and miR150 target c-myb and result in altered megakaryocyte-erythroid cell fate (Grabher et al., 2011).

In the present study, we identified miR-199b-5p as a positive erythroid regulator, that was regulated by the key erythroid transcription factors GATA-1 and NF-E2. The up-regulation of miR-199b-5p during erythroid differentiation was dependent on the binding of GATA-1 and NF-E2 to its gene locus, which activated its transcription and maintained its high expression level in mature erythroid cells. Moreover, miR-199b-5p directly repressed c-Kit expression to promote erythroid maturation. In brief, our study successfully identified erythroid miR-199b-5p as an important regulator of human erythropoiesis.

Received 15 October, 2013; revised 28 December, 2013; accepted 30 December, 2013; published online 6 March, 2014

Keywords: c-Kit, erythroid differentiation, GATA-1, microRNA-199b-5p, NF-E2 
GATA-1 and NF-E2 Up-Regulate miR-199b-5p in Erythropoiesis Yuxia Li et al.

\section{MATERIALS AND METHODS}

\section{Cell lines and cell culture}

K562 (human chronic myelogenous leukemia cell line) was maintained in DMEM supplementing with $10 \%$ fetal bovine serum (GIBCO, USA).

To induce of erythroid differentiation in K562 cells, $30 \mu \mathrm{M}$ hemin (Sigma-Aldrich, Germany) was added to the medium for the duration of the experiment. Benzidin staining was used to detect hemoglobin-positive cells. 293T cells were obtained from American Type Culture Collection and were cultured in DMEM media with $10 \%$ FBS.

\section{Oligonucleotides and transfection}

MiRNA-199b-5p mimic, miRNA-199b-5p inhibitor and negative control molecules were obtained from Dharmacon (USA) and transfected into K562 cells at a final concentration of $60 \mathrm{nM}$. The K562 cells were washed the next day with PBS and plated for induction using hemin. siRNAs smart pools (specifically for GATA-1 or NF-E2) and control siRNA pools were synthesized by Dharmacon and transfected into K562 cells (100 nM) using the Neon ${ }^{\circledR}$ Transfection System (Invitrogen, USA). The medium was replaced after $6 \mathrm{~h}$, and the cells were cultured for 48 $\mathrm{h}$ and harvested for Western blot analyses as described below.

\section{RNA extraction and quantitative real-time PCR}

Total RNA was extracted from the harvested cells using Trizol reagent (Invitrogen, USA) according to the manufacturer's instructions. The RNA was quantified based on its absorbance at $260 \mathrm{~nm}$. cDNA was synthesized using M-MLV reverse transcriptase (Invitrogen) from $2 \mu \mathrm{g}$ of total RNA.

Oligo- (dT)-18 was used as the RT primers for the reverse transcription of mRNAs. Stem-loop RT primers were used for the reverse transcription of miRNAs. Quantitative RT-PCR was performed using the ABI PRISM 7500 real-time PCR System (Applied Biosystems, USA) using the SYBR Premix ExTaq kit (Takara, China) according to the manufacturer's instructions. For mRNAs, the data were normalized using endogenous GAPDH as a control. For miRNAs, U6 snRNA was used as the endogenous control. The comparative Ct method was used to quantify target genes relative to their endogenous control. For each individual analysis, one of the samples was designated as the calibrator and assigned a relative value of 1.0. All of the quantities were detected at $0 \mathrm{~h}, 24 \mathrm{~h}, 48 \mathrm{~h}$ and $72 \mathrm{~h}$ posttransfection as described below.

\section{Flow cytometry}

The K562 cells were harvested at the indicated times and washed twice at $4^{\circ} \mathrm{C}$ in PBS/0.5\% BSA to block Fc receptors. The Cells were incubated with PE-conjugated anti-CD71 and FITC-conjugated anti-CD235a antibodies for 30 min (eBioscience, $1 \mu \mathrm{g} / \mathrm{ml}$ ). Flow cytometric data measuring PI fluorescence were acquired from approximately $10^{5}$ cells using a C6 (BD) flow cytometer. All assays were carried out in triplicate.

Chromatin immuneprecipitation (ChIP) assay

The antibodies anti-GATA-1 (ab11963, Abcam Company) AntiNF-E2 (sc365083, Santa Cruz Biotechnology) and anti -Pol II (ab5408, Abcam Company), were used for the ChIP assays. K562 cells induced using hemin for the indicated time points were collected and cross-linked with $1 \%$ formaldehyde for 10 min, washed in cold PBS buffer, resuspended in lysis buffer [0.1\% SDS, 0.5\% Triton X-100, $20 \mathrm{mM}$ Tris- $\mathrm{HCl}(\mathrm{pH} 8.1), 150$ $\mathrm{mM} \mathrm{NaCl}$, protease inhibitor, (Roche)], and sonicated to obtain chromatin fragments between $200 \mathrm{bp}$ and 1,000 bp in length. The sonicated chromatin was resuspended in IP buffer and incubated overnight at $4^{\circ} \mathrm{C}$ with magnetic beads conjugated antibodies (Santa Cruz Biotechnologies). The IP was then washed with lysis buffer, $\mathrm{LiCl}$ buffer $(0.25 \mathrm{M} \mathrm{LiCl}, 1 \% \mathrm{NP}-40$, $1 \%$ deoxycholate, $1 \mathrm{mM}$ EDTA, $10 \mathrm{mM}$ Tris- $\mathrm{HCl}[\mathrm{pH} 8.1])$ and TE buffer, eluted in elution buffer ( $1 \%$ SDS, $0.1 \mathrm{M} \mathrm{NaHCO}_{3}$ ). The DNA was then recovered by reversing the crosslinks, and purified by QIAGEN Purification Kit. An un-enriched sample of DNA was treated in a similar manner to serve as [input].

\section{Western blot analysis}

Whole-cell lysates or nuclear extracts were subjected to Western blot analysis as detailed previously (Yu et al., 2008). The following antibodies were used for Western blot. The GAPDH antibody was purchased from Santa Cruz Biotechnology. NFE2 (ABE413) was purchased from Millipore Company. GATA-1 (ab11963) was purchased from Abcam.

\section{Statistics}

Student's $t$-test (two-tailed) was performed to analyze the data. $P$-values $<0.05$ were to be considered significantly different, as indicated by an asterisk ( ${ }^{\star} P$-values $<0.05 ;{ }^{\star \star} P$-values $<0.01$ ).

\section{RESULTS}

MiR-199b-5p is up-regulated during human erythropoiesis Previously, our study screening for potential GATA-1-activated erythroid miRNAs indicated miR-199b-5p as a candidate, as well as other potential candidate miRNAs including miR-652, -154, -193b, -375, -145 and -148 (Zhu et al., 2013) (Fig. 1A). Following this study, we performed another quantitative RTPCR (q-RT-PCR) analysis to characterize the expression of these selected miRNAs in K562 cells undergoing erythropoiesis via hemin treatment. It was found that miR-199b-5p expression was continuously increased during K562 erythropoiesis (Fig. 1B), while other miRNAs also exhibited up-regulated expression after hemin treatment (Fig. 1B). A previous study revealed the aberrantly high expression of miR-199b-5p in polycythemia vera (PV), a myeloproliferative disorder (MPD) of the blood in which the bone marrow produces too many red blood cells. Because miR-199b-5p appeared to be up-regulated in maturing erythroid cells, we hypothesized that miR-199b-5p may function to promote erythroid maturation. Taken together, these findings suggested that miR-199b-5p might act as a positive modulator of human erythropoiesis.

\section{Erythroid transcription factors GATA-1 and NF-E2 reside and activate miR-199b-5p expression}

GATA-1 is an essential hematopoietic transcription factor that participates in the expression of numerous genes involved in erythropoiesis. GATA-1-null mice exhibited a complete ablation of erythropoiesis due to the arrested maturation and apoptosis of the erythroid precursors at the proerythroblast stage. Another transcription factor NF-E2 is also crucial for regulation of erythroid-specific gene expression. The expression of globin genes in developing erythroid cells is controlled by upstream locus control regions. The activation of these regions in vivo requires NF-E2 binding.

As erythropoiesis proceeds, the levels of GATA-1 and NF-E2 both increased (Fig. 2A), which was consistent with the expression pattern of miR-199b-5p (Fig. 1B), suggesting a association between the regulation of these two transcription factors and miRNAs. In order to examine whether GATA-1 and NF-E2 


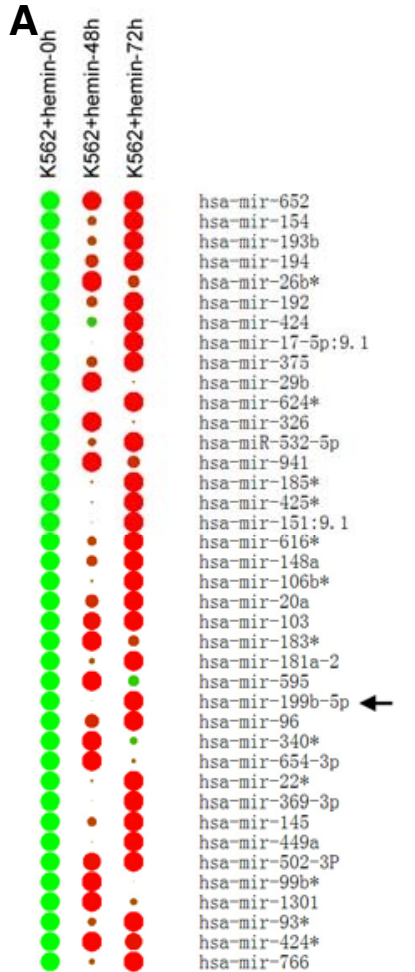

B
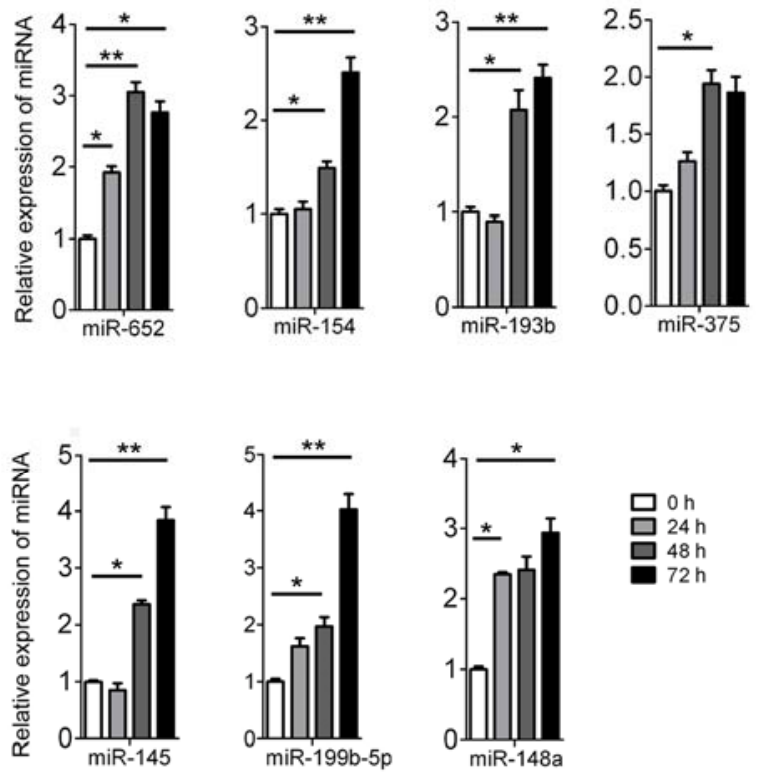

Fig. 1. Validation of miRNA expression during K562 erythroid differentiation. (A) The schematic diagram illustrating the up-regulated miRNAs during K562 erythropoiesis. (B) Real-time PCR analysis of the expression of selected miRNAs in hemin-treated $\mathrm{K} 562$ cells at $0 \mathrm{~h}, 24 \mathrm{~h}, 48 \mathrm{~h}$ and $72 \mathrm{~h}$. The error bars represent the standard deviation obtained from three independent experiments. ${ }^{*} p<$ $0.05 ;{ }^{* *} p<0.01$.

위 구 으오으 잉

affect miR-199b-5p expression in erythroid cells, we treated K562 cells with either DNA constructs expressing GATA-1/NFE2 or siRNAs specific to GATA-1/NF-E2 to achieve the overexpression or knock-down of transcription factors (Fig. 2B). The results of Western blot analysis indicated that GATA-1 was overexpreesed $\sim 3$-fold in pcDNA-GATA-1 transfected K562 cells compared to the empty vector-treated cells. Inversely, the GATA-1 expression level was reduced to $\sim 40 \%$ upon siRNAs treatment. Similarly, NF-E2 expression increased $\sim 4$-fold in overexpression assay and decreased $\sim 2$-fold in the knockdown experiment. Accordingly, miR-199b-5p increased in both GATA-1 and NF-E2 overexpressing K562 cells, whereas it was inhibited in GATA-1 and NF-E2 knock-down K562 cells (Fig. 2C). These results indicated that the expression of miR-199b$5 p$ was controlled by GATA-1 and NF-E2.

Furthermore, in our attempt to investigate the potential binding sites of GATA-1 and NF-E2, a Transcription Element Search System (TESS, http://www.cbil.upenn.edu/cgi-bin/tess) -mediated sequence analysis was performed and revealed putative GATA-1 and NF-E2 binding sites scattered within the upstream region of the human miR-199b locus (Fig. 3A). ChIP-PCR analysis was used to validate the binding and showed that both GATA-1 and NF-E2 occupied upstream of miR-199b-5p in $48 \mathrm{~h}$ hemin-treated K562 cells (Fig. 3B). Taken together, these results indicated that erythroid transcription factors GATA-1 and NF-E2 resided and activated miR-199b-5p expression in erythroid cells.

MiR-199b-5p promotes erythroid differentiation of K562 cells To examine whether miR-199b-5p affect erythroid differentiation, we transfected a miR-199b-5p mimic and a negative con- trol into K562 cells. These K562 cells were then induced to undergo erythroid differentiation via hemin treatment. The influence of K562 cell erythroid differentiation was detected with benzidine (DAB) staining, fluorescence activated cell sorting (FACS) and q-PCR analysis respectively. The results of DAB staining conformed that miR-199b-5p overexpression (Fig. 4A) increased the proportion of benzidine-positive K562 cells after hemin treatment (Figs. 4B and 4C).

Moreover, gamma-globin expression was clearly up-regulated in miR-199b-5p overexpressing K562 cells compared to the negative control at 48h (Fig. 4D; Supplementary Fig. S1A). To further test and verify our finding, we analyzed the expression of two major erythroid cell surface markers (CD71/CD235a) with FACS. The overexpression of miR-199b-5p raised the percentage of CD71+/CD235a+ cells significatively compared to the negative control (Fig. 4E). In contrast, the inhibition of endogenous miR-199b-5p using miRNA inhibitors markedly repressed the erythroid differentiation of K562 cells (Fig. 4F; Supplementary Fig. S1B), as supported by decreased percentage of CD71+/ CD235a+ cells (Fig. 4G), and reduced gamma-globin expression (Supplementary Figs. S1C and S1D) compared to the negative control. Taken together, these results demonstrated that miR-199b-5p promoted erythroid differentiation in K562 cells.

MiR-199b-5p represses c-Kit to regulate erythropoiesis MiRNAs regulate specific mRNA targets by interfering their stability or repressing their translation. Therefore, we used the TargetScan and PicTar algorithms to obtain potential mRNAs containing miR-199b-5p-binding sites. MRNA targets related to erythropoiesis and hit by both algorithms were considered as candidates and were subjected to immunoblotting assays (Fig. 
A K562 induced by hemin
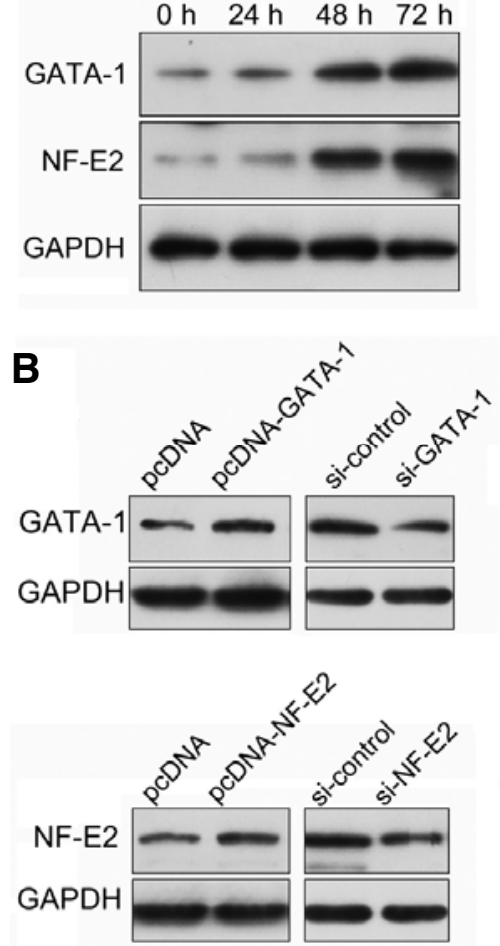

C

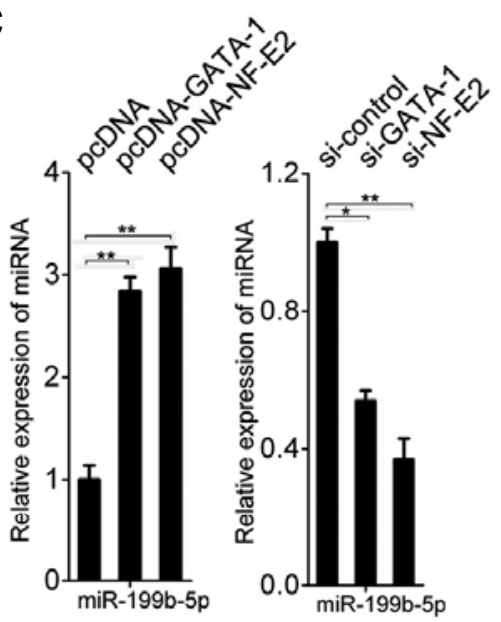

Fig. 2. Identification of GATA-1/NF-E2activated miR-199b-5p in K562 cells. (A) Western blot revealing the levels of GATA-1 and NF-E2 were both increased in hemin-treated $\mathrm{K} 562$ cells. The signals are normalized to GAPDH. (B) Western blot analysis of K562 cells transfected with a pcDNA construct overexpressing GATA1/NF-E2 or siRNAs specific to either GATA-1/NF-E2 to achieve the overexpression or knock-down of these transcription factors. (C) Real-time PCR analysis of miR-199b-5p expression inK562 cells with GATA-1/ NF-E2 overexpression or knockdown.

A
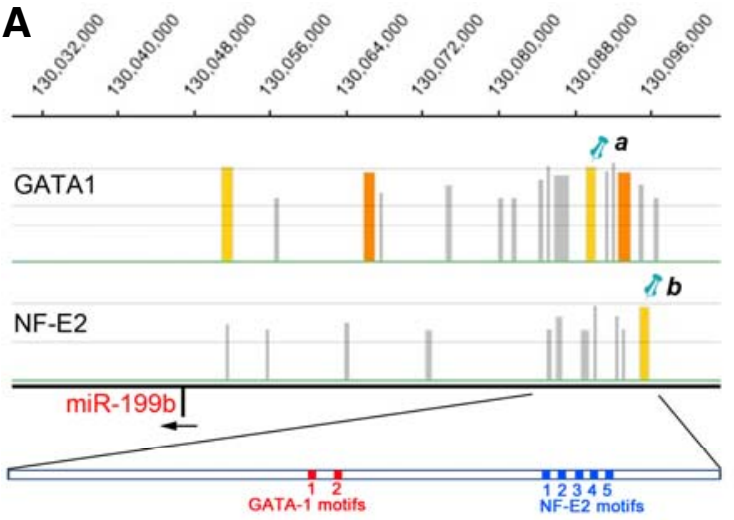

B

\begin{tabular}{|l|c|c|c|c|c|}
\hline \multirow{2}{*}{ MicroRNA } & \multirow{2}{*}{ Peak ID } & \multirow{2}{*}{ Positive motif } & \multicolumn{3}{|c|}{ ChIP-PCR results } \\
\cline { 4 - 5 } & & & input & IgG & GATA \\
\hline miR-199b & \multirow{2}{*}{11388} & motif 2 & & \\
\hline
\end{tabular}

\begin{tabular}{|c|c|c|c|c|c|}
\hline \multirow{2}{*}{ MicroRNA } & \multirow{2}{*}{ Peak ID } & \multirow{2}{*}{ Positive motif } & \multicolumn{3}{|c|}{ ChIP-PCR results } \\
\hline & & & input & $\lg G$ & NF-E2 \\
\hline \multirow{3}{*}{ miR-199b } & \multirow{3}{*}{7586} & motif 1 & 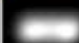 & & \\
\hline & & motif 3 & & & 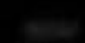 \\
\hline & & motif 4-5 &  & & 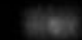 \\
\hline
\end{tabular}

peak a

chr9: $130089180-130090129$

peak $b$

chr9: 130094713 - 130095762
Fig. 3. GATA-1 and NF-E2 were located on the upstream of miR-199 and activated its expression during erythropoiesis. (A) Representation of the GATA-1 and NF-E2 motifs upstream of the human miR-199 locus. (B) ChIP-PCR analysis of the GATA-1 and NF-E2 hits upstream of the miR-199 in K562 cells. 
A

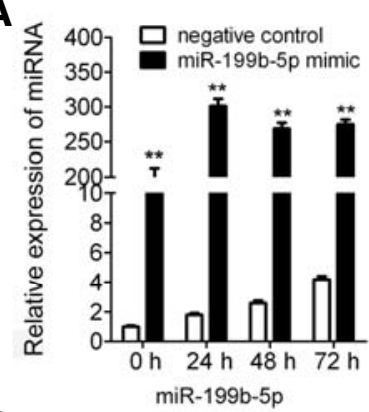

C

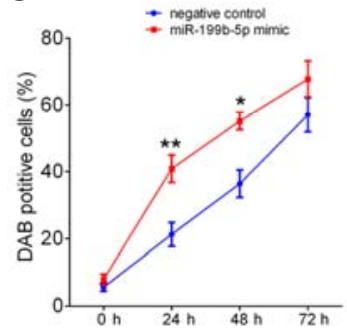

B

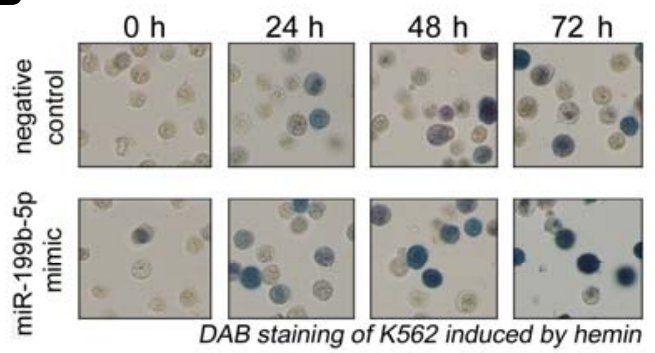

D

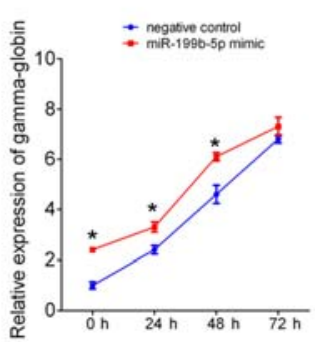

E

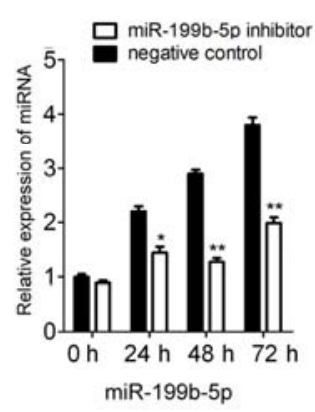

E



G



Fig. 4. MiR-199b-5p promotes erythroid differentiation of $\mathrm{K} 562$ cells. (A) Real-time PCR analysis of the expression of miR-199b-5p in K562 cells transfected with miR-199b-5p mimic or negative control. ${ }^{*} p<0.05$; ${ }^{* *} p<0.01$. (B, C) DAB staining of miR-199b-5p overexpression increased the proportion of benzidine-positive cells in K562 cells after $0 \mathrm{~h}, 24 \mathrm{~h}$, $48 \mathrm{~h}$, and $72 \mathrm{~h}$ of hemin treatment. Representative benzidine staining of K562 cells was shown in (B). ${ }^{*} p<0.05 ;{ }^{* *} p<0.01$. (D) Real-time analysis of gammaglobin expression in miR-199b-5p mimic-treated K562 cells after 0 $\mathrm{h}, 24 \mathrm{~h}, 48 \mathrm{~h}$, and $72 \mathrm{~h}$ hemin induction. (E) FACS analysis of K562 cells following transfection with miR-199b-5p mimic or negative control and hemin induction for $24 \mathrm{~h}$. (F) Real-time PCR analysis of the expression of miR199b-5p in K562 cells transfected with miR-199b-5p inhibitor or negative control. ${ }^{*} p<0.05$; ${ }^{*} p<$ 0.01. (G) FACS analysis of K562 cells following transfection with miR-199b-5p inhibitor or negative control and hemin induction for 0 $\mathrm{h}$ and $48 \mathrm{~h}$.
5A; Supplementary Table S2). Besides, c-Kit was predicted to be a potential target of miR-199b-5p by TargetScan (Fig. 5A). The stem cell factor receptor (SCF) c-Kit plays a vital role in modulating cell proliferation and survival in various cell types. In particular, c-Kit is obligatory for the early amplification of erythroid progenitors. However, as the erythroid cell enters in maturation stage, c-Kit disappears from the cell surface (D'Allard et al., 2013). Therefore, although c-Kit was only predicted by the TargetScan algorithm, its role in erythroid differentiation made it an additional candidate that was examined in subsequent assays (Fig. 5A). To confirm the regulatory interactions between miR-199b-5p and its potential targets, we evaluated the effects of miR-199b-5p overexpression on the endogenous target protein levels in K562 cells. As expected, the ectopic expression of miR-199b-5p in K562 cells reduced c-Kit, TAF9B and CCNL1 expression levels by $\sim 2$-fold, $\sim 3$-fold and $\sim 4$-fold, respectively, compared to the control (Fig. 5B), whereas the expression levels of GRB10 and NLK were not changed after miR-199b-5p overexpression. Thus, our attempt to investigate the regulatory mechanism of miR-199b-5p during erythropoiesis led to the identification of its potential target genes, including c-Kit, TAF9B and CCNL1.

\section{DISCUSSION}

MicroRNAs are negative regulators of gene expression that have garnered great interest due to their role as post-transcriptional regulators of genes involved in numerous physiologic and developmental processes (Heuston et al., 2011). Differential miRNA expression is largely controlled by various transcription factors. In our previous studies, for example, miR-223 reversibly regulates the erythroid and megakaryocytic differentiation of K562 cells via down-modulation of LMO2. miR-376 down-regulates $A g o 2$ and $C D K 2$ which inhibit progenitor cell growth and differentiation (Wang et al., 2011). MiR-23a is a critical erythroid miRNA gene that not only is a positive regulator of erythroid differentiation but is also activated by GATA-1. In this study, we chose a potential erythroid-associated miRNA, miR-199b-5p to validate its expression, function, regulation and mechanism during erythroid differentiation. MiR-199b-5p was up-regulated during K562 erythropoiesis. Two erythroid transcription factors GATA-1 and NF-E2 occupied the upstream of miR-199b locus and activated its transcription. Moreover, miR-199b-5p could promote erythroid differentiation by repressing c-Kit expression in K562 cells. 
GATA-1 and NF-E2 Up-Regulate miR-199b-5p in Erythropoiesis

Yuxia Li et al.

\begin{tabular}{|c|c|c|c|c|c|}
\hline $\begin{array}{l}\text { Target prediction } \\
\text { algorithm }\end{array}$ & $\begin{array}{l}\text { Target } \\
\text { gene }\end{array}$ & Full name of target gene & $\begin{array}{l}\text { Position in } \\
\text { 3'UTR }\end{array}$ & \multicolumn{2}{|r|}{$\begin{array}{c}\text { Complementary sites between miR-199b-5p } \\
\text { and target genes }\end{array}$} \\
\hline TargetScan & KIT & $\begin{array}{l}\text { v-kit Hardy-Zuckerman } 4 \\
\text { feline sarcoma viral } \\
\text { oncogene bomolog }\end{array}$ & $1652-1658$ & $5^{\prime}$ & $\begin{array}{l}\text { GGAAGUAACCAUUUGCACUGGAG } \\
\qquad|||| \mid \\
\text { CUUGUCUAUCAGAUUUGUGACCC }\end{array}$ \\
\hline TargetScan/PicTar & GRB10 & $\begin{array}{l}\text { Growth factor receptor-bound } \\
\text { protein } 10\end{array}$ & $39-46$ & $5^{\prime}$ & $\begin{array}{l}\text { GACUGGAGGAAGUGA--ACACUGGA } \\
\text { CUUGUCUAUCAGAUUUGUGACCC }\end{array}$ \\
\hline TargetScan/PicTar & TAF9B & $\begin{array}{l}\text { TAF9B RNA polymerase II, } \\
\text { TATA box binding protein } \\
\text { (TBP)-associated factor, } 31 \mathrm{kDa}\end{array}$ & $347-954$ & $5^{\prime}$ & $\begin{array}{l}\text { UUUUGAUAAGUAAUUACACUGGA } \\
\qquad|||\quad||||| \mid \\
\text { CUUGUCUAUCAGAUUUGUGACCC }\end{array}$ \\
\hline TargetScan/PicTar & NLK & Nemo-like kinase & $43-49$ & $5^{\prime}$ & $\begin{array}{l}\text { UGUAAUGUAGCUUUC-CACUGGAG } \\
\qquad|||||||||| \\
\text { CUUGUCUAUCAGAUUUGUGACCC }\end{array}$ \\
\hline TargetScan/PicTar & CCNL1 & Cyclin L1 & $378-384$ & $5^{\prime}$ & $\begin{array}{l}\text { GUGAAUACUUUCUUAACACUGGU } \\
\qquad|||||| \mid \\
\text { CUUGUCUAUCAGAUUUGUGACCC }\end{array}$ \\
\hline
\end{tabular}

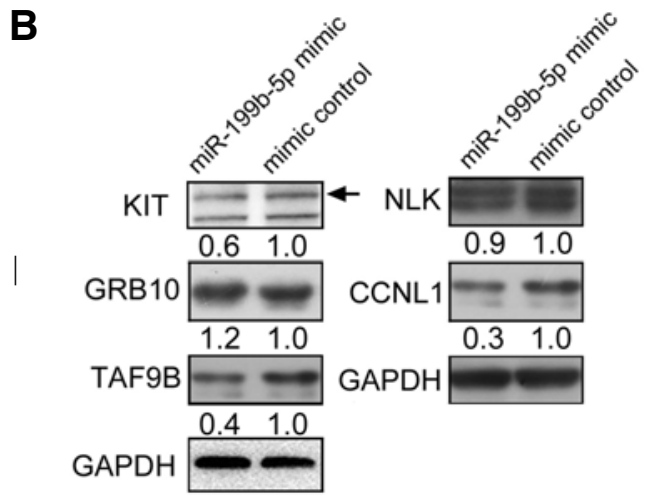

MiR-199b-5p has been discussed in various biological systems, including chronic myeloid leukemia, medulloblastoma, renal cell carcinoma and colorectal cancers (Andolfo et al., 2012; Flamant et al., 2010; Garzia et al., 2009; Wu et al., 2012). In different tissue types, miR-199b-5p acts distinct activities and is coordinated with specific transcription factors (D'Allard et al., 2012; Fang et al., 2013). One study by Bruchova et al. (2008) revealed that miR-199b expression was markedly increased in patients suffering from PV compared to healthy controls. PV is a clonal myeloproliferative disorder (MPD) caused by somatic mutations that arise in a hematopoietic multipotent cell. Hematopoiesis in PV is characterized by the accumulation of phenotypically normal erythrocytes. Thus, our finding of accumulated miR-199b-5p in matured erythroid cells was in consistent with the PV phenotype, which also suggesting miR-199b-5p as a potential therapy target in PV treatment.

Note: Supplementary information is available on the Molecules and Cells website (www.molcells.org).

\section{ACKNOWLEDGMENTS}

This work was supported by grants from the National Natural Science Foundation of China [31040021 to J.Y., 31200977 to F. W.]; the foundation for Key Teacher by Henan University (G201122).
Fig. 5. MiR-199b-5p represses c-Kit, TAF9B and CCNL1 expression to regulate erythropoiesis. (A) Computer prediction of miRNA-binding sites in the $3^{\prime}$ UTR of potential target genes. (B) Western blot analysis of c-Kit, TAF9B, CCNL1, GRB10 and NLK in K562 cells treated with miR-199b-5p mimic or negative control.

\section{REFERENCES}

Andolfo, I., Liguori, L., De, A.P., Cusanelli, E., Marinaro, F., Pistollato, F., Garzia, L., De, V.G., Petrosino, G., Accordi, B., et al. (2012). The micro-RNA 199b-5p regulatory circuit involves Hes1, CD15, and epigenetic modifications in medulloblastoma. Neuro Oncol. 14, 596-612.

Azzouzi, I., Moest, H., Winkler, J., Fauchère, J.C., Gerber, A.P., Wollscheid, B., Stoffel, M., Schmugge, M., and Speer, O. (2011). MicroRNA-96 directly inhibits c-Globin expression in human erythropoiesis. PLoS One 6, e22838.

Bellon, M., Lepelletier, Y., Hermine, O., and Nicot, C. (2009). Deregulation of microRNA involved in hematopoiesis and the immune response in HTLV-I adult T-cell leukemia. Blood 113, 4914-4917.

Bousquet, M., Harris, M.H., Zhou, B.Y., and Lodish, H.F. (2010). MicroRNA miR-125b causes leukemia. Proc. Natl. Acad. Sci. USA 107, 21558-21563.

Bruchova, H., Merkerova, M., and Prchal, J.T. (2008). Aberrant expression of microRNA in polycythemia vera. Haematologica 93, 1009-1016.

D’Allard, D., Gay, J., Descarpentries, C., Frisan, E., Adam, K., Verdier, F., Floquet, C., Dubreuil, P., Lacombe, C., Fontenay, M., et al. (2013). Tyrosine kinase inhibitors induce down-regulation of c-kit by targeting the ATP pocket. PLoS One 8, e60961.

Fang, C., Zhao, Y., and Guo, B. (2013). MiR-199b-5p targets HER2 in breast cancer cells. J. Cell. Biochem. 114, 1457-63.

Faraoni, I., Laterza, S., Ardiri, D., Ciardi, C., Fazi, F., and Lo-Coco, F. (2012). MiR-424 and miR-155 deregulated expression in cytogenetically normal acute myeloid leukaemia: correlation with 
NPM1 and FLT3 mutation status. J. Hematol. Oncol. 5, 26.

Flamant, S., Ritchie, W., Guilhot, J., Holst, J., Bonnet, M.L., Chomel, J.C., Guilhot, F., Turhan, A.G., and Rasko, J.E. (2010). MicroRNA response to imatinib mesylate in patients with chronic myeloid leukemia. Haematologica 95, 1325-1333.

Fukao, T., Fukuda, Y., Kiga, K., Sharif, J., Hino, K., Enomoto, Y., Kawamura, A., Nakamura, K., Takeuchi, T., and Tanabe, M. (2007). An evolutionarily conserved mechanism for microRNA223 expression revealed by microRNA gene profiling. Cell 129, 617-631.

Garzia, L., Andolfo, I., Cusanelli, E., Marino, N., Petrosino, G., Martino, D.D., Esposito, V., Galeone, A., Navas, L., and Esposito, S. (2009). MicroRNA-199b-5p impairs cancer stem cells through negative regulation of HES1 in medulloblastoma. PLoS One 4, e4998.

Grabher, C., Payne, E.M., Johnston, A.B., Bolli, N., Lechman, E., Dick, J.E., Kanki, J.P., and Look, A.T. (2011). Zebrafish microRNA-126 determines hematopoietic cell fate through c-Myb. Leukemia 25, 506-514.

Heuston, E.F., Lemon, K.T., and Arceci. R.J. (2011). The beginning of the road for non-coding RNAs innormal hematopoiesis and hematologic malignancies. Front Genet. 2, 94

Huang, X., Gschweng, E., Van, H.B., Cheng, D., Mikkola, H.K., and Witte, O.N. (2011). Regulated expression of microRNAs-126/ $126^{*}$ inhibits erythropoiesis from human embryonic stem cell. Blood 117, 2157-2165.

Navarro, F., and Lieberman, J. (2010). Small RNAs Guide Hematopoietic Cell Differentiation and Function. J. Immunol. 184, 5939-
5947.

Pase, L., Layton, J.E., Kloosterman, W.P., Carradice, D., Waterhouse, P.M., and Lieschke, G.J. (2009). miR-451 regulates zebrafish erythroid maturation in vivo via its target gata2. Blood 113, 1794-1804.

Wang, F., Yu, J., Yang, G.H., Wang, X.S., and Zhang, J.W. (2011). Regulation of erythroid differentiation by miR-376a and its targets. Cell Res. 21, 1196-1209.

Wu, X.W., Weng, L.H., Li, X.J., Guo, C., Pal, S.K., Jin, J.M., Li, Y.P., Nelson, R.A., Mu, B., Onami, S.H., et al. (2012). Identification of a 4-microRNA Signature for clear cell renal cell carcinoma metastasis and prognosis. PLoS One 7, e35661.

Yu, J., Ryan, D.G., Getsios, S., Oliveira-Fernandes, M., Fatima, A and Lavker, R.M. (2008). MicroRNA-184 antagonizes microRNA-205 to maintain SHIP2 levels in epithelia. Proc. Natl. Acad. Sci. USA 105, 19300-19305.

Yu, D., dos Santos, C.O., Zhao, G., Jiang, J., Amigo, J.D., Khandros, E., Dore, L.C., Yao, Y., D'Souza, J., Zhang, Z., et al. (2010). miR-451 protects against erythroid oxidant stress by repressing 14-3-3zeta. Genes Dev. 24, 1620-1633.

Zhang, L.B., Flygare, J., Wong, P., Lim, B., and Lodish, HF. (2010). miR-191 regulates mouse erythrob last enucleation by downregulating Riok3 and Mxi1. Genes Dev. 25, 119-124.

Zhu, Y., Wang, D.S., Wang, F., Li, T.T., Dong, L., Liu, H.W., Ma, Y.N., Jiang, F.B., Yin, H.X., Yan, W.T., et al. (2013). A comprehensive analysis of GATA-1-regulated miRNAs reveals miR-23a to be a positive modulator of erythropoiesis. Nucleic Acids Res. $41,4129-4143$ 